\title{
The study to estimate the floating population in Seoul, Korea
}

\author{
Geon Woo Lee ${ }^{1}$, Yong Jin Lee ${ }^{1}$, Youngeun $\mathrm{Kim}^{1}$, Seung-Han Hong ${ }^{1}$, Soohwaun Kim ${ }^{1}$, \\ Jeong Soo Kim², Jong Tae Lee ${ }^{2}$, Dong Chun Shin ${ }^{3}$, Youngwook Lim ${ }^{1}$ \\ ${ }^{1}$ Institute for Environmental Research, Yonsei University College of Medicine, Seoul, Korea; ${ }^{2}$ Transportation Pollution Research \\ Center, National Institute of Environmental Research, Incheon, Korea; ${ }^{3}$ Department of Preventive Medicine, Yonsei University \\ College of Medicine, Seoul, Korea
}

Traffic-related pollutants have been reported to increase the morbidity of respiratory diseases. In order to apply management policies related to motor vehicles, studies of the floating population living in cities are important. The rate of metro rail transit system use by passengers residing in Seoul is about $54 \%$ of total public transportation use. Through the rate of metro use, the people-flow ratios in each administrative area were calculated. By applying a people-flow ratio based on the official census count, the floating population in 25 regions was calculated. The reduced level of deaths among the floating population in 14 regions having the roadside monitoring station was calculated as assuming a $20 \%$ reduction of mobile emission based on the policy. The hourly floating population size was calculated by applying the hourly population ratio to the regional population size as specified in the official census count. The number of people moving from 5 a.m. to next day 1 a.m. could not be precisely calculated when the population size was applied, but no issue was observed that would trigger a sizable shift in the rate of population change. The three patterns of increase, decrease, and no change of population in work hours were analyzed. When the concentration of particulate matter less than $10 \mu \mathrm{m}$ in aerodynamic diameter was reduced by $20 \%$, the number of excess deaths varied according to the difference of the floating population. The effective establishment of directions to manage the pollutants in cities should be carried out by considering the floating population. Although the number of people using the metro system is only an estimate, this disadvantage was supplemented by calculating inflow and outflow ratio of metro users per time in the total floating population in each region. Especially, 54\% of metro usage in public transport causes high reliability in application.

Keywords Floating population, Seoul, Metro rider, Population inflow, Population outflow

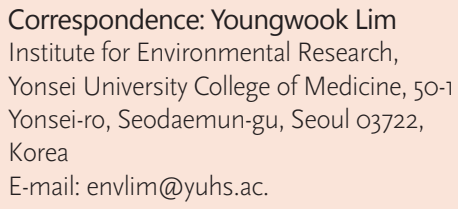

Correspondence: Youngwook Lim Institute for Environmental Research, Yonsei University College of Medicine, 50-1 Yonsei-ro, Seodaemun-gu, Seoul 03722, Korea

E-mail: envlim@yuhs.ac.

Received: March 7, 2017 Accepted: May 15, 2017 Published: May 26, 2017

This article is available from: http://e-eht.org/

\section{INTRODUCTION}

Traffic-related air pollutants in cities are reported by many studies as causing respiratory diseases, including asthma, as well as increasing the number of deaths from cardiovascular diseases or lung cancer [1-3].

It has been discovered by many studies that inhalation of fine dust, one of the main emissions from mobile sources, may in- crease the risk of death from underlying diseases in the heart and lungs as its concentration level rises [4]. The contaminants emitted from such mobile sources may vary in concentration depending on the regions. In particular, people may have a higher chance of being exposed to such contaminants when riding an automobile and a bicycle or walking in the cities [5]. The city of Seoul, which is composed of 25 administrative districts, is also conducting a study with the objective of ensuring policy- 
level protection from contaminants by estimating the number of people exposed to contaminants. Control and oversight are being provided, however, using population statistics calculated from the official census count. The floating populations by region and time, however, are quite dissimilar. Their exposure to environmental contaminants is closely related to their activity pattern per unit of time [6], the regional floating population change, and their activity pattern. To expedite the introduction of a policy such as control of automobile entry by area and time, it is all the more important to study the floating populations by area and time in the city of Seoul.

In this study, the changing trend in the floating population by area in Seoul was determined by checking the turnover rate through an analysis of the number of metro riders. For this purpose, the floating population is defined as the number of people at a given point in time who are in transit from one place to another.

\section{METHODS}

\section{Calculation of the Floating Population in Seoul}

Objective data related to the population flow are required to calculate the size of the floating population in Seoul, but no such data on the interregional people flow with the required accuracy is available, due to some practical issues. Manhattan, one of biggest cities in the US, has analyzed its public transport usage status and calculated the floating population [7]. The usage rate of the metro (subway) system in Manhattan was similar to that of Seoul. Therefore, the number of riders by station throughout the 282 metro stations in Seoul was analyzed. The number of subway stations in each area is as follows. The numbers of subway stations in Gangnam-gu, Gangdong-gu, Gangbuk-gu, Gangseogu, Gwanak-gu, Gwangjin-gu, Guro-gu, Geumcheon-gu, Nowon-gu, Dobong-gu, Dongdaemun-gu, Dongjag-gu, Mapo-gu, Seodaemun-gu, Seongdong-gu, Seocho-gu, Songpa-gu, Seongbuk-gu, Yeongdeungpo-gu, Yongsan-gu, Jongno-gu, Jung-gu, Yangcheon-gu, Eunpyeong-gu, and Jungrang-gu are 22, 10, 4, 19, $4,10,7,1,11,3,6,16,15,5,14,15,22,9,16,10,15,22,6,13$, and 7, respectively. The metro riders account for about $54 \%$ of the total public transport riders in Seoul, thereby making it a challenge to calculate the exact interregional moving trend [8], but it was determined that the number of such riders could be applicable if their flow rate was to be analyzed (Table 1).

The information on the Seoul metro lines that are currently in service was analyzed by collecting information from metro lines $\# 1$ to \#4 as well as from the KORAIL lines and from metro lines $\# 5$ to \#8 and metro line \#9. Some data were collected from the 2011 data, which contains information on the 282 stations, excluding a few stations that do not provide the relevant data.

The authors confirmed the locations of stations by administrative district before checking the hourly number of riders getting on and off the trains at each station. The hourly size of the floating population in each region was calculated by converting the hourly number of riders getting on and off the trains as compared to the total number of daily riders getting on and off the trains in the stations across the 25 wards into their respective ratios. For the hours when the metros are not running, it was assumed that the residents of each region are staying in their respective regions from 1 to 4 a.m., setting the population as specified in the resident registry as the basis for calculation.

\section{Calculation of Excess Deaths Due to Exposure to $\mathrm{PM}_{10}$}

To estimate the number of excess deaths due to exposure to particulate matter less than $10 \mu \mathrm{m}$ in aerodynamic diameter $\left(\mathrm{PM}_{10}\right)$ for this analysis, the log-linear C-R function used by Lee et al. [9] was employed.

$\left.\Delta \mathrm{N}=\mathrm{Y}_{0}(\exp (-\beta \Delta \mathrm{P})-1)\right)^{*} \mathrm{Pop}$

$\Delta \mathrm{N}$ : Number of excess deaths after $20 \%$ reduction of $\mathrm{PM}_{10}$

$\mathrm{Y}_{0}$ : Number of excess deaths before $20 \%$ reduction of $\mathrm{PM}_{10}$

$\beta$ : Proportional constant $(=$ in $[$ relative risk $] / \Delta \mathrm{PM})$

$\Delta \mathrm{P}$ : Variation in the concentration of the contaminants

Pop: Population

The relative risk for the fine dust and nitrogen dioxide adopted in this study was calculated based on overseas study results, as local study results are not available $[10,11]$. Assuming a $20 \%$ reduction in emissions, we estimated the number of deaths that

Table 1. Average use of public transport on weekdays in the metropolitan area in 2012

\begin{tabular}{|c|c|c|c|c|c|}
\hline \multirow{2}{*}{ Region } & \multirow{2}{*}{$\begin{array}{c}\text { Total of bus and metro } \\
\text { users (n) }\end{array}$} & \multicolumn{3}{|c|}{ Bus } & \multirow{2}{*}{ Metro } \\
\hline & & Express & City & Small & \\
\hline Total & 20421806 & $854469(4.2)$ & $9591788(47.0)$ & 2488967 (12.2) & $7486582(36.7)$ \\
\hline Seoul & 10544507 & $308008(2.9)$ & 4205683 (39.9) & 305037 (2.9) & 5725779 (54.3) \\
\hline Incheon & 1101862 & $16011(1.5)$ & 483539 (43.9) & 196794 (17.9) & 405518 (36.8) \\
\hline Gyeonggi & 6120978 & 519419 (8.5) & $3487847(57.0)$ & 807977 (13.2) & 1305735 (21.3) \\
\hline The others ${ }^{\mathrm{a}}$ & 2654459 & $11031(0.4)$ & 1414719 (53.3) & $1179159(44.4)$ & $49550(1.9)$ \\
\hline
\end{tabular}

Values are presented as number (\%).

The others are areas that are not in the metropolitan area. 
could be prevented. $\mathrm{PM}_{10}$ concentration was determined by using data collected from roadside monitoring stations.

\section{RESULTS}

\section{Calculation of Population Based on the Number of Metro Riders}

The default value for the calculation of the floating population was based on the population within the respective official census count per district as specified in the resident registry, while the population as of 2011 was assumed as the base year for the analysis of the number of metro riders.

To calculate the hourly change ratio based on the number of metro users, a database was prepared by classifying the riders along the regions where the 282 metro stations were located. Because stations were located in each region, it was judged that the reliability of the data would be improved by estimating the floating population through the calculation of their respective ratios. The limitation of these data is that some of the relevant information, including the number of people traveling to and from the other satellite cities in Gyeonggi-do, or to and from Incheon, could not be verified. Still, it was concluded that a 54\% usage rate would be sufficient for verifying the ratio of the overall population change by region. The hourly population ratio for
20 hours during the operation hours of the metro system from 5 to 1 a.m. was calculated, and the population size was calculated by applying the hourly population ratio to the regional population as specified in the official census count to estimate the hourly floating population (Table 2). The number of people moving from 5 a.m. to the following 1 a.m. could not be precisely calculated when the population size was applied, so the estimations are of necessity imprecise, but no problem was observed that would have triggered a significant shift in the population change rate. The imprecision is understood to have stemmed from the impact of the non-returning population or from the outgoing or inflowing population from the other regions. The number of people during the period from 1 to 5 a.m., when the metro is not operating, was set based on the number of residents specified in the resident registry and assumed to be staying in the given region.

The calculation of the regional floating population showed three main population flow patterns. Type A showed that the floating population increased during work hours, but decreased during the after-work hours; this pattern was found in the districts of Gangnam-gu, Geumcheon-gu, Mapo-gu, Seodaemungu, Seocho-gu, Yeongdeungpo-gu, Yongsan-gu, Jongno-gu, and Jung-gu. Type B had a population decrease compared to the usual number, as people migrated to the type $\mathrm{B}$ regions, with the

Table 2. The ratio of the average change in the number of daily passengers on weekdays on the metro in 2012

\begin{tabular}{|c|c|c|c|c|c|c|c|c|c|c|c|c|c|c|c|c|c|c|c|c|}
\hline \multirow{2}{*}{ Region (gu) } & \multicolumn{20}{|c|}{ Changes in the ratio of the number of metro passengers ( \%) } \\
\hline & $5-6$ & $6-7$ & $7-8$ & $8-9$ & $9-10$ & $10-11$ & $11-12$ & $12-13$ & $13-14$ & $14-15$ & $15-16$ & $16-17$ & $17-18$ & $18-19$ & $19-20$ & $20-21$ & $21-22$ & $22-23$ & $23-24$ & $24-1$ \\
\hline angnam & -0.3 & 1.3 & 5.1 & 19.5 & 31 & 3.9 & 1.6 & 0.6 & 0.4 & -0.4 & -1.6 & -2.0 & -3.7 & -12.0 & -8.2 & -5.1 & -5.6 & -4.8 & -1.6 & 0.0 \\
\hline & 3 & -2.1 & -8.7 & & & & & & & & & 1.1 & & & & & & & & 1.2 \\
\hline & 4 & 3.6 & -12.6 & -9.0 & & & & & & & & & & & & & & & & .1 \\
\hline & & -2.0 & -8.9 & -8.2 & -3.2 & -2.0 & & & & & & & & & & & & & & .1 \\
\hline Nallak & 8 & -2.9 & -11.1 & -12.4 & -3 & & & & & & & & & & & & & & & .8 \\
\hline & & -0.9 & -4.5 & -3.7 & -0 & & & & & & & & & & & & & & & 0.9 \\
\hline & 2 & -2.6 & -7.5 & -0.8 & & & & & & & & & & & & & & & & .5 \\
\hline & 2 & 1.2 & & & & & & & & & & & & & & & & & & .0 \\
\hline ow & & -2.9 & -10.1 & -7.2 & -2 & & & & & & & & & & & & & & & .5 \\
\hline & & -3.9 & -16.0 & -10.8 & -2.6 & & & & & & & & & & & & & & & .7 \\
\hline & 8 & -1.1 & -4.4 & & & & & & & & & & & & & & & & & .8 \\
\hline & & -1.4 & -6. & & & & & & & & & & & & & & & & & .0 \\
\hline apo & -0.6 & -0.2 & -1.7 & & & & & & & & & & & & & & & & & 0.4 \\
\hline & -0.9 & -0.8 & 0.4 & 12.9 & & & -0. & & -0 & & -0 . & -0 & -1. & -11 & -2.4 & -1 & -1.2 & 0.8 & & 0.6 \\
\hline ons & & 1.7 & & & & & & & & & & & -2 & & & & -3.7 & -3.2 & & 0.3 \\
\hline & & & & & & & & & & & & & & & & & & & & 1.0 \\
\hline & & -1.6 & -6.5 & -3.5 & -0.7 & -0.4 & -0. & -0.7 & -0. & -0. & & -0.1 & -0.4 & & 4.7 & & & & & .1 \\
\hline & -0.6 & -0.1 & -3.4 & & & & & & & & & & -1.2 & & & & & & & 0.9 \\
\hline Yangcheon & -0.9 & -2.0 & -7.9 & -4.9 & -0.2 & -0.6 & -0.6 & -0 & & & 0. & & 0.6 & 2. & 2 & & 1.5 & & & 0.9 \\
\hline & .8 & 0.1 & & & & & & & & & -0. & -2 & -6.5 & & -2 & -2 & -1.6 & 0.0 & & .4 \\
\hline & & 0.5 & & & & & & & & & -0.2 & -1.0 & -1.9 & -2.3 & & -1.7 & -3.1 & -3.7 & -1.0 & 0.4 \\
\hline Eunpyeong & -1.4 & -2.3 & -8.9 & -7.4 & -2.9 & -1.9 & -1.6 & -1.3 & -1.2 & -0.5 & & 0.8 & 1.1 & 3.9 & 6.6 & 4.1 & 3.6 & 4.1 & 3.6 & 1.4 \\
\hline Jongno & -0.4 & 1.2 & 5.3 & & & 3.1 & 2 & 1. & & & & -2.9 & -4.5 & -10.5 & -4.9 & -4.4 & -5.1 & -3.6 & -0.9 & 0.1 \\
\hline & -0.7 & 0.7 & 3.4 & 15.4 & 7.1 & 2.6 & 0.9 & 0.1 & & -0.4 & -1.5 & -2.1 & -3.5 & -8.5 & -4.4 & -3.2 & -3.3 & -2.9 & -0.1 & 0.2 \\
\hline Jungnang & -1.8 & -2.5 & -9.5 & -9.3 & -4.5 & -2.2 & -1.6 & -1.4 & -1.0 & -0.3 & 0.7 & 1.4 & 1.8 & 4.7 & 7.3 & 4.5 & 4.1 & 4.5 & 3.6 & 1.4 \\
\hline
\end{tabular}


applicable districts being Gangdong-gu, Gangbuk-gu, Gangseogu, Gwanak-gu, Gwangjin-gu, Guro-gu, Nowon-gu, Dobonggu, Seongdong-gu, Seongbuk-gu, Yangcheon-gu, Eunpyeong$\mathrm{gu}$, and Jungrang-gu. Type $\mathrm{C}$ showed that the population inflow-outflow trend was not stable or sustained; this pattern characterized Dongdaemun-gu and Songpa-gu. In the case of types $\mathrm{A}$ and $\mathrm{B}$, the extent of the population inflow and outflow varied significantly by region. The population increase was significant during work hours in Gangnam-gu, Geumcheon-gu, Jongno-gu, and Jung-gu compared to the other districts, whereas the population decrease was pronounced in Dobong-gu, Gangbu-gu, Gwanak-gu, Eunpyeong-gu, Gangseo-gu, and Nowon-gu. Such changes in the flow pattern are believed to be closely related to the number of businesses or homes per area. In the case of the type $\mathrm{C}$ districts, such as Dongdaemun-gu and Songpa-gu, the population inflow and outflow were similar in different hours, suggesting that their living patterns are somewhat different from those in the other districts. The study had limitations, however, in classifying the indoor or outdoor population or the number of people by subregion. Such data could have been derived by applying the indoor/outdoor activity hours provided by the National Statistical Office, but it would have been a stretch to apply the data to this study.

\section{Calculation of the Number of Deaths That Can Be Prevented With the Reduction of $\mathrm{PM}_{10}$}

The number of possible annual deaths was estimated by applying the resident population specified in the resident registry. The number of possible annual deaths due to $\mathrm{PM}_{10}$ was determined to be 1233 across the 14 districts where there are roadside monitoring stations, including 24 in Jung-gu, 43 in Yongsan-gu, 35 in Jongno-gu, and 83 in Dongdaemun-gu.

When the number of mobile sources was reduced by $20 \%$, the number of excess deaths among those specified in the resident registry that can be prevented was 15 in Jung-gu and 33 in Yongsan-gu. The number of excess deaths that can be prevented can either drop or rise depending on the change in the hourly floating population. The results of this study do not suggest that the deaths are occurring by the hour based on the number of excess deaths that can be prevented, but that the traffic policy should be applied differently depending on the size of the floating population.

\section{DISCUSSION}

The results of the floating population in this study can be confirmed by checking the number of economically active people per district, suggesting that the data obtained will be useful for policy application with the hourly population trends.

This study has the following limitations: (1) the different activities of the floating population, depending on indoor or outdoor presence, were not confirmed; and (2) interregional movement by other public transport systems besides the metro system could not be considered. However, the disadvantages were offset by calculating the metro's inflow and outflow ratio of people per unit of time in relation to the total floating population of each region. In particular, a rate of 54\% of metro usage in public transport makes for a high degree of reliability in application.

The Seoul Metropolitan Government provides the density of workers to a given population by surveying the density of workers and population per region [12]. The density of workers per population was highest in Jung-gu (262.7) according to this study, followed by Jongno-u (131.1), Gangnam-gu (110.8), Seocho-gu (89.9), and Geumcheon-gu (75.0), suggesting a density higher than the average level, while that of Eunpyeonggu was 15.8; that of Nowon-gu and Dobong-gu, 17.1 for each; that of Gangbuk-gu, 19.6; and that of Gwanak-gu, 19.9. The density of workers per given population was similar to patterns of types A and B in the results of this study, suggesting that the application of the hourly ratio to metro riders in the calculation of the floating population is plausible.

The number of excess deaths was calculated based on the scenario in which people are continuously exposed to the concentration levels measured by the roadside measurement network for one year. Somewhat different results are expected if the indoor space or interregional migration will be counted in as well. Given that the hazard inflicted on the human body by mobile sources as well as the need to reduce such contaminants was confirmed, however, the study results are believed to be helpful for policy application.

Continued studies should be conducted to ensure the minimization of the exposure to contaminants, while a more effective policy that considers the floating population should be adopted.

\section{ACKNOWLEDGEMENTS}

This work was carried out with the support of "Health Risk Assessment for Population Exposure Due to Vehicle Emission" of the Korea Automobile Environmental Association, Republic of Korea.

\section{CONFLICT OF INTEREST}

The authors have no conflicts of interest associated with the material presented in this paper. 


\section{ORCID}

Geon Woo Lee https://orcid.org/0000-0002-3344-0411

Yong Jin Lee https://orcid.org/0000-0002-7859-2611

Youngeun Kim https://orcid.org/0000-0002-9195-0831

Seung-Han Hong https://orcid.org/0000-0002-4675-3580

Soohwaun Kim https://orcid.org/0000-0002-8553-3628

Dong Chun Shin https://orcid.org/0000-0002-3055-9935

Youngwook Lim https://orcid.org/0000-0002-2420-4943

\section{REFERENCES}

1. Atkinson RW, Anderson HR, Sunyer J, Ayres J, Baccini M, Vonk $\mathrm{JM}$, et al. Acute effects of particulate air pollution on respiratory admissions: results from APHEA 2 project. Air Pollution and Health: a European Approach. Am J Respir Crit Care Med 2001; 164(10 Pt 1):1860-1866.

2. Dominici F, Peng RD, Bell ML, Pham L, McDermott A, Zeger SL, et al. Fine particulate air pollution and hospital admission for cardiovascular and respiratory diseases. JAMA 2006;295(10):11271134.

3. Zanobetti A, Schwartz J, Dockery DW. Airborne particles are a risk factor for hospital admissions for heart and lung disease. Environ Health Perspect 2000;108(11):1071-1077.

4. Wilson R, Spengler J, editors. Particles in our air: concentrations and health effects. Cambridge: Harvard School of Public Health;
1996, p. 169-188.

5. McCreanor J, Cullinan P, Nieuwenhuijsen MJ, Stewart-Evans J, Malliarou E, Jarup L, et al. Respiratory effects of exposure to diesel traffic in persons with asthma. N Engl J Med 2007;357(23):23482358.

6. National Institute of Environmental Research. The study of individual exposure assessment according to pattern of daily activity time. Incheon: National Institute of Environmental Research; 2009, p. 264-266 (Korean).

7. Moss ML, Qing C. The dynamic population of manhattan; 2012 [cited 2017 May 25]. Available from: https://wagner.nyu.edu/ rudincenter/publication/the-dynamic-population-of-manhattan/.

8. Korea Railroad Corporation. The forecast for demand of long-term rail transport in 2013. Daejeon: Pooreun AD; 2013, p. 63 (Korean).

9. Lee YJ, Lim YW, Yang JY, Kim CS, Shin YC, Shin DC. Evaluating the PM damage cost due to urban air pollution and vehicle emissions in Seoul, Korea. J Environ Manage 2011;92(3):603-609.

10. Samet JM, Dominici F, Zeger SL, Schwartz J, Dockery DW. The National Morbidity, Mortality, and Air Pollution Study. Part I: methods and methodologic issues. Res Rep Health Eff Inst 2000; (94 Pt 1):5-14.

11. Sunyer J, Castellsagué J, Sáez M, Tobias A, Antó JM. Air pollution and mortality in Barcelona. J Epidemiol Community Health 1996; 50 Suppl 1:s76-s80.

12. Seoul Metropolitan Government. Workers density table [cited 2017 May 31]. Available from: http://data.seoul.go.kr/openinf/ linkview.jsp?infId=OA-11744 (Korean). 